\title{
Länderfinanzausgleich 2020: Neue Form - alte Probleme
}

\author{
Der neue Länderfinanzausgleich wird erstmals 2020 praktiziert. Die Analyse seiner \\ Funktionsweise und Wirkungen zeigt, dass gegenüber dem alten System große formale, aber \\ nur geringe materielle Unterschiede bestehen. Die Reform hat vorhandene Defizite sogar noch \\ verschärft. Der Autor schlägt eine Modifikation des Länderfinanzausgleichs vor, die auf eine \\ Verbesserung der finanziellen Anreizeffekte zielt und die Grenzbelastungen deutlich reduziert.
}

Die Bundesländer verfügen über Steuereinnahmen, die sich nach dem Prinzip des örtlichen Aufkommens auf die Länder verteilen. Dazu gehören die Länderanteile an den Gemeinschaftsteuern ohne Umsatzsteuer sowie die Ländersteuern im engeren Sinne (z.B. Erbschaftsteuer, Grunderwerbsteuer). Die originäre Steuerkraft bildet das Fundament des horizontalen Länderfinanzausgleichs. Seine Aufgabe besteht darin, alle Länder finanziell so auszustatten, dass sie ihre verfassungsmäßigen Aufgaben erfüllen können. Da die Wirtschafts- und Steuerkraft der Länder enorme Unterschiede aufweist, bedarf es hierzu einer Angleichung ihrer Finanzkraft.

\section{Horizontale Verteilung der Umsatzsteuer}

Der Länderfinanzausgleich erfolgt in mehreren Stufen. Auf der ersten Stufe wird der Länderanteil an der Umsatzsteuer verteilt. Bis 2019 erhielten steuerschwache Länder bis zu $25 \%$ der Umsatzsteuer der Länder als Ergänzungsanteile. Der Rest wurde nach Einwohnern verteilt. Die Auffüllung durch Ergänzungsanteile orientierte sich an der relativen Steuerkraft und sorgte für eine kräftige Nivellierung der Steuerkraftpositionen nach Umsatzsteuerzerlegung. Während sich die für 2020 geschätzte Steuerkraft der Länder vor Umsatzsteuer zwischen $56 \%$ (ST) und $156 \%(\mathrm{HH})$ des Bundesdurchschnitts bewegt, würden die entsprechenden Werte mit Umsatzsteuervorwegausgleich zwischen $93 \%$ und $129 \%$ liegen. $^{1}$

(C) Der/die Autor(en) 2020. Open Access: Dieser Artikel wird unter der Creative Commons Namensnennung 4.0 International Lizenz (https:// creativecommons.org/licenses/by/4.0/deed.de) veröffentlicht.

Open Access wird durch die ZBW - Leibniz-Informationszentrum Wirtschaft gefördert.

1 Verwendet werden die üblichen Ländercodes (https://t1p.de/z43h9). Quantitative Angaben beruhen auf einer Simulation des Länderfinanzausgleichs auf der Basis der Daten des BMF (2020). Das Steueraufkommen ist aufgrund der absehbaren Rezession zu hoch angesetzt, doch ändert das nichts an den strukturellen Systemeigenschaften.
Ab 2020 entfallen die Ergänzungsanteile und alle Bundesländer erhalten gleiche Beträge pro Einwohner. Auch bei diesem Verteilungsmaßstab ergibt sich eine deutliche Verminderung der Steuerkraftdifferenzen auf $73 \%$ bis $134 \%$. Gegenüber dem alten System profitieren neben den relativ steuerstarken auch zwei relativ steuerschwache Länder (RP, NW), weil die Ergänzungsanteile die nach Einwohnern verteilten Mittel schmälern und dadurch im Grenzbereich Ausgleichsbedürftigkeit erst erzeugen.

Die Pro-Kopf-Steuerkraft aller Länder erhöht sich 2020 um denselben absoluten Umsatzsteuerbetrag von 1.420 Euro pro Einwohner (vgl. Abbildung 1). Gemessen an der Pro-Kopf-Steuerkraft liegen die ostdeutschen Flächenländer und das Saarland erheblich unter dem Bundesdurchschnitt von 3.661 Euro pro Einwohner. Berlin erzielt einen leicht überdurchschnittlichen Wert und gehört damit (neben BW, BY, HE und $\mathrm{HH}$ ) zu den relativ steuerstarken Ländern.

Dass die Nivellierungseffekte der Umsatzsteuerverteilung deutlich kleiner ausfallen als bisher, ist per Saldo kein distributiver Mangel. Zum einen orientiert sich die Umsatzsteuerverteilung an der relativen Steuerkraft, während auf den folgenden Stufen mit der relativen Finanzkraft ein stark abweichender Maßstab verwendet wird. Zum anderen bestimmen die Ausgleichseffekte auf dieser Stufe nur über die Ausgangspositionen auf den weiteren Stufen und werden dort entscheidend korrigiert; nach der Reform noch wesentlich stärker als zuvor.

Prof. Dr. Wolfgang Scherf ist Inhaber der Professur für Öffentliche Finanzen - VWL II an der Justus-Liebig-Universität Gießen. 
Abbildung 1

Horizontale Verteilung der Umsatzsteuer

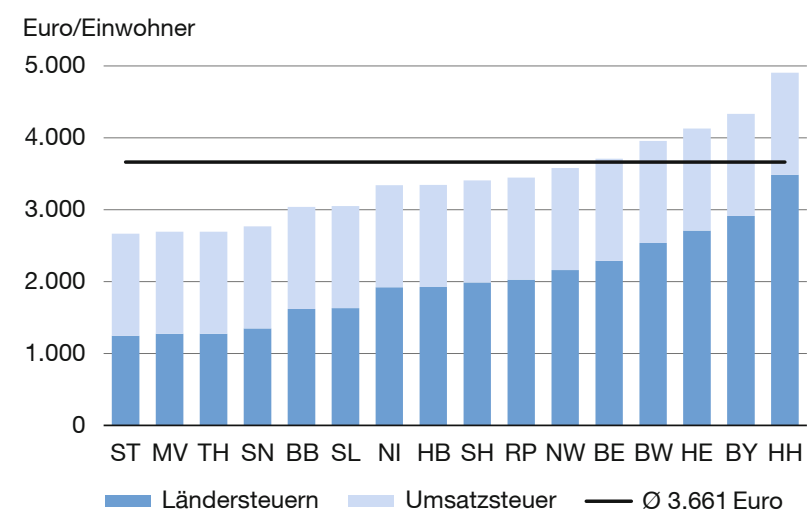

Quelle: eigene Darstellung.

\section{Finanzkraftausgleich unter den Ländern}

Auf der zweiten Stufe existierte bis 2019 ein horizontaler Finanzkraftausgleich unter den Ländern. Dabei zahlten relativ reiche Länder Ausgleichsbeiträge, mit denen symmetrische Ausgleichzuweisungen an relativ finanzschwache Länder finanziert wurden. Formal entfällt der direkte Ausgleich ab 2020, aber er wurde vollwertig durch finanzkraftabhängige Zuschläge und Abschläge ersetzt, die mit den Umsatzsteueranteilen verrechnet werden. Die Ausgleichseffekte auf der zweiten Stufe hängen nicht mehr von der relativen Steuerkraft, sondern von der relativen Finanzkraft ab. Die Finanzkraftmesszahl unterscheidet sich von der Steuerkraftmesszahl durch den anteiligen Einbezug der Gemeindesteuern. Sie rechneten bis 2019 mit einem Anteil von 64 \% zur Finanzkraft der Länder. Ab 2020 steigt die Anrechnungsquote auf $75 \%$. Abbildung 2 sortiert die Länder nach ihrer originären Steuerkraft und zeigt die Erhöhung der Finanzkraft gegenüber der Ländersteuerkraft pro Einwohner durch Anrechnung der Gemeindesteuern. Die mittlere Finanzkraftmesszahl liegt bei 4.718 Euro pro Einwohner. Der Wechsel von der relativen Steuer- zur Finanzkraft als Ausgleichsmaßstab ist entscheidend für die Ausgleichseffekte auf der zweiten Stufe. Die Zu- und Abschläge bei der Umsatzsteuer orientieren sich am Verhältnis zwischen der Finanzkraftmesszahl (FMZ) und der Ausgleichsmesszahl (AMZ). Letztere entspricht nicht der durchschnittlichen Steuerkraft pro tatsächlichem Einwohner, sondern basiert auf gewichteten Einwohnern. Daraus resultieren erhebliche Abweichungen zwischen der relativen Steuer- und Finanzkraft der Bundesländer.

Die Einwohnerwertungen bei der Ausgleichsmesszahl wurden nicht verändert. Gegenüber den Einwohnern der anderen Länder werden die Einwohner der Stadtstaaten
Abbildung 2
Von der Steuerkraft zur Finanzkraft

Euro/Einwohner

7.000

6.000

5.000

4.000

3.000

2.000

1.000

0

ST MV TH SN BB SL NI HB SH RP NW BE BW HE BY HH

$$
\begin{aligned}
& \text { Ländersteuern } \quad \text { Umsatzsteuer } \\
& \text { Gemeindesteuern } \quad \varnothing 4.718 \text { Euro }
\end{aligned}
$$

Quelle: eigene Darstellung.

bei den Länder- und Gemeindesteuern weiterhin mit $135 \%$ deutlich und die Einwohner der dünn besiedelten Flächenländer bei den Gemeindesteuern leicht höher gewichtet (ST $102 \%$, BB $103 \%$, MV $105 \%$ ). Die Ausgleichsmesszahl der Stadtstaaten steigt gegenüber dem Durchschnitt um 1.486 Euro pro Einwohner, während die zu tatsächlichen Einwohnern gewerteten Flächenländer eine Verminderung um 122 Euro pro Einwohner hinnehmen müssen. Entsprechend stark profitieren die Stadtstaaten im Finanzausgleich von der Vergrößerung inrer Bedarfsmesszahlen. ${ }^{2}$

Der horizontale Länderfinanzausgleich wird weiterhin auf der Basis der Finanzkraft- und Ausgleichsmesszahlen durchgeführt. Dabei gilt ein einheitlicher Ausgleichssatz von $63 \%$. Abbildung 3 ordnet die Länder nach der Finanzkraftmesszahl und illustriert die Verteilungseffekte des neuen Systems. Infolge der Stadtstaatenwertung ist Bremen gemessen an der FMZ-AMZ-Relation von $72 \%$ das relativ ärmste Bundesland. Auch Berlin rechnet mit $75 \%$ zur Gruppe der besonders finanzschwachen Länder. Deutlich über dem Durchschnitt liegen nur Bayern, Hessen, BadenWürttemberg und Hamburg. Auch auf der zweiten Stufe des Länderfinanzausgleichs erfolgt eine starke Nivellierung. Die Finanzkraftspanne sinkt nach Verrechnung der Zu- und Abschläge bei der Umsatzsteuer auf $90 \%$ (HB) bis $108 \%$ (BY). Gegenüber dem alten System hat sich die Nivellierungsintensität deutlich vermindert. Das geht weniger auf den linearen Ausgleichstarif zurück, dessen Wirkungen auf die relati-

2 Die Einwohnerwertung, die alle anderen Länder belastet, bildet positive Wirkungen der Stadtstaaten auf angrenzende Flächenländer nicht korrekt ab. Die Zerlegung der Lohnsteuer nach Arbeitsorten (Weiß und Münzenmaier, 2014) oder die Zerlegung der Gemeinschaftssteuern nach der Bruttowertschöpfung (Glinka und Lenk, 2015) würde das Stadt-Umland-Problem besser lösen. 
Abbildung 3

Finanzkraftausgleich unter den Ländern

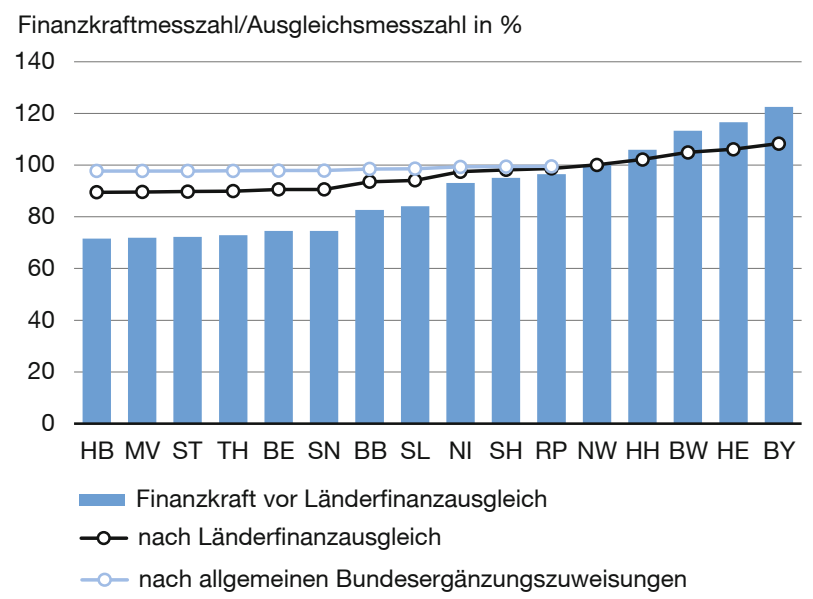

Quelle: eigene Darstellung.

ve Finanzkraft weitgehend denen des alten Progressionstarifs entsprechen. Die Ursache liegt vielmehr in den größeren Finanzkraftdifferenzen, die bei Verteilung der Umsatzsteuer nach Einwohnern zunächst verbleiben. Aus distributiver Sicht ist der schwächere Ausgleichseffekt unproblematisch, denn er wird durch die allgemeinen Bundesergänzungszuweisungen auf der nächsten Stufe korrigiert.

\section{Bundesergänzungszuweisungen}

Abbildung 3 zeigt auch den Effekt der allgemeinen Bundesergänzungszuweisungen. Sie setzen den horizontalen Länderfinanzausgleich mit einem vertikalen Instrument fort. Die relativ finanzschwachen Länder erhalten zusätzliche Bundesmittel zur Deckung ihres Finanzbedarfs. Ab 2020 werden Fehlbeträge bis 99,75\% (2019: 99,5\%) des Länderdurchschnitts zu 80 \% (2019: 77,5\%) ausgeglichen. Damit erhöht sich die Ausgleichsintensität so stark, dass finanzschwache Länder im alten wie im neuen System fast identische Relationen zwischen Finanzkraft- und Ausgleichsmesszahl erreichen. Die Finanzkraftspanne verkleinert sich noch einmal deutlich auf rund $98 \%$ bis $108 \%$.

Die Tendenz zur Vertikalisierung des Finanzausgleichs (Heinemann, 2016) zeigt sich auch bei den Bundesergänzungszuweisungen für Sonderbedarfe. Hier wurden neue Ausgleichsansprüche etabliert. Wenn die Gemeindesteuern pro Einwohner unter $80 \%$ des Durchschnitts liegen, erhalten die Länder nun spezielle Zuweisungen, mit denen die Fehlbeträge zum Durchschnitt zu 53,5\% ausgeglichen werden. Es ist gewiss kein Zufall, dass diese Zuweisungen den ostdeutschen Flächenländern und sonst nur dem Saarland zugute kommen. Die früheren Ergänzungszuweisungen für teilungsbedingte Sonderlasten wurden unter anderem mit

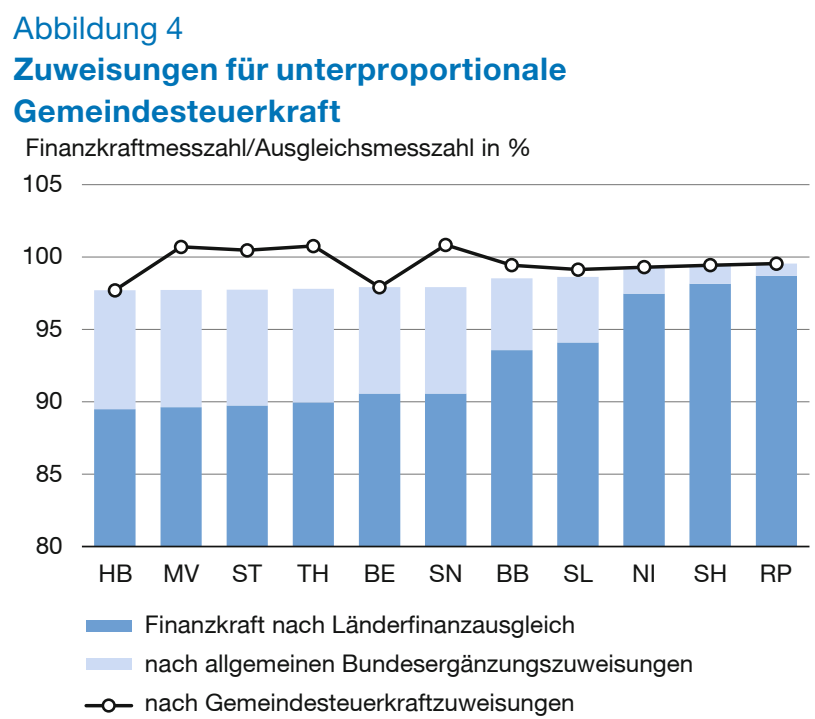

Quelle: eigene Darstellung.

der geringen kommunalen Finanzkraft begründet. Augenscheinlich wird der Solidarpakt mit den Gemeindesteuerkraftzuweisungen teilweise fortgesetzt.

Die Funktion dieser Ergänzungszuweisungen im Gesamtsystem ist kaum zu begründen. Das gemeinsame Potenzial der Länder und Gemeinden zur Finanzierung öffentlicher Aufgaben und Ausgaben hängt nicht von der Struktur, sondern vom Niveau der gesamten Finanzkraft ab. Dennoch erhält von zwei Ländern mit insgesamt gleicher Finanzkraft nun ein Land mit unterproportionaler Gemeindesteuerkraft Ergänzungszuweisungen, während das andere leer ausgeht. Das Land mit unterproportionaler Gemeindesteuerkraft wird also besser gestellt, obwohl seine Finanzkraft keine besondere Bedarfslage signalisiert. Daher wäre es folgerichtig, auf diese Bundesergänzungszuweisungen zu verzichten, die frei werdenden Bundesmittel via Umsatzsteueranteil an die Länder auszuschütten und auf der dadurch erhöhten Basis den allgemeinen Finanzkraftausgleich wirken zu lassen.

Andernfalls ergeben sich unsystematische Ausgleichswirkungen, die eine Übernivellierung bewirken können. Abbildung 4 zeigt, dass Länder, die Gemeindesteuerkraftzuweisungen erhalten, andere Länder mit zuvor höherer Finanzkraft nicht nur einholen, sondern klar überholen. Das gilt selbst innerhalb der Gruppe der Empfängerländer. So liegt Mecklenburg-Vorpommern nach allgemeinen Bundesergänzungszuweisungen hinter, nach Gemeindesteuerkraftzuweisungen jedoch vor Sachsen-Anhalt. Gleiches gilt für die Länder Sachsen, Brandenburg und Saarland, deren Reihenfolge vertauscht wird. Obwohl die Zuweisungen formal unabhängig von den Maßstäben des Finanzkraftausgleichs gewährt werden, dürften sie materiell gegen das verfassungsrechtliche Übernivellierungsverbot verstoßen. 
Abbildung 5

Gesamteffekt des Länderfinanzausgleichs

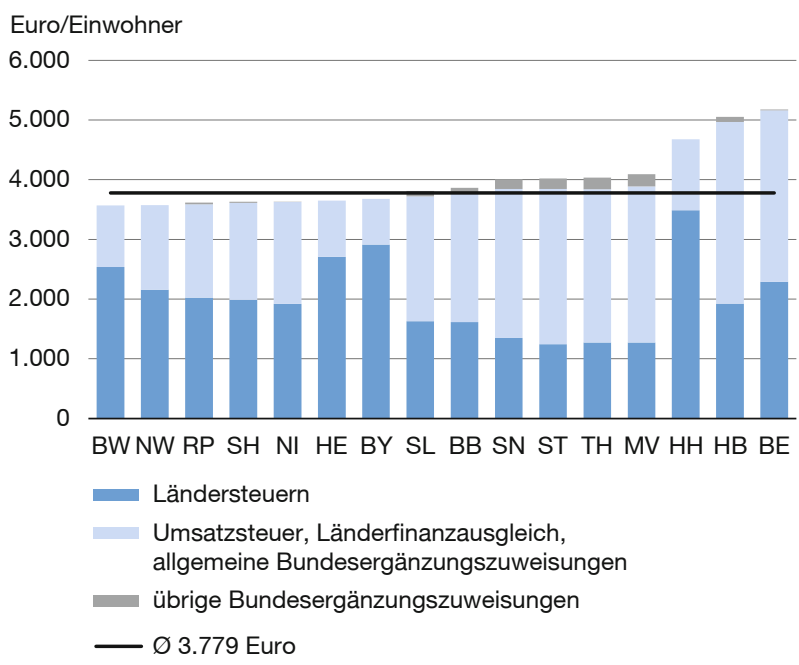

Quelle: eigene Darstellung.

Eine weitere Neuerung sind Bundesergänzungszuweisungen zum Ausgleich unterproportionaler Mittel aus der Forschungsförderung. Wenn der Nettozufluss pro Einwohner unter $95 \%$ des Durchschnitts liegt, werden die Fehlbeträge zu $35 \%$ kompensiert. Die Maßnahme ist ein gutes Beispiel für die fragliche Dotierung vermeintlicher Sonderbedarfe im Finanzausgleich. Bei der Forschungsförderung liegt gar kein Sonderbedarf vor, sondern nur eine unterproportionale Zuteilung von Bundesmitteln für einen Zweck, der nichts mit dem Finanzausgleich zu tun hat.

\section{Gesamteffekt des Länderfinanzausgleichs}

Im Grunde ist der neue Länderfinanzausgleich der alte geblieben. Der horizontale Ausgleich bleibt in Form finanzkraftbezogener Zu- und Abschläge bei der Umsatzsteuer erhalten. Die reduzierten Ausgleichseffekte durch die Umsatzsteuerverteilung nach Einwohnern und den proportionalen Ausgleichstarif werden durch eine erhebliche Ausweitung der Bundesergänzungszuweisungen nachgeholt. Finanziell profitieren die Länder zulasten des Bundes von einer Reform, die an den Verteilungseffekten des Länderfinanzausgleichs wenig geändert hat.

Das Gesamtergebnis der Umverteilung lässt sich anhand der effektiven Pro-Kopf-Einnahmen der Länder präzisieren (vgl. Abbildung 5). Bis auf Hamburg weisen Länder mit überdurchschnittlicher Steuerkraft vor Finanzausgleich (blaue Säulen) nach Umsatzsteuerverteilung, horizontalem Länderfinanzausgleich und allgemeinen Bundesergänzungszuweisungen (hellblaue Säulen) eine unterdurchschnittliche Finanzkraft auf. Die westdeutschen Flächenländer liegen klar unter dem Durchschnitt, während die ostdeutschen
Flächenländer inn knapp erreichen oder übertreffen. In der Finanzkraftrangfolge führen mit großem Abstand die Stadtstaaten. Insbesondere Berlin und Bremen verbessern ihre Position aufgrund der Einwohnerwertung. Inklusive der übrigen Bundesergänzungszuweisungen für Sonderbedarfe überschreiten alle ostdeutschen Länder aufgrund der Zuweisungen für unterproportionale Gemeindesteuerkraft klar den Durchschnittswert. Auch Bremen und Berlin profitieren davon und können ihre Spitzenposition weiter ausbauen. Dagegen rutscht z. B. das steuerstarke Bundesland Hessen in der Länderrangfolge von Platz 3 vor Finanzausgleich auf Platz 11 nach Finanzausgleich ab.

\section{Anreizfeindliche Grenzbelastungen der Länder}

Der neue Länderfinanzausgleich hat weiterhin eine enorm hohe Nivellierungsintensität. Das Problem der ausgeprägten Umverteilung besteht vor allem in den hohen Grenzbelastungen, die bei einem Anstieg der Steuereinnahmen eines Landes auftreten. Sie erzeugen negative Anreizeffekte, weil sich Anstrengungen zur Verbesserung der eigenen Wirtschafts- und Steuerkraft finanziell für ein Bundesland kaum auszahlen. Die Länder tragen zwar die Kosten ihrer Aktivitäten, müssen aber die daraus resultierenden Steuereinnahmen zu einem großen Teil an die anderen Bundesländer und an den Bund abführen. Alle Länder verlieren durch geringere Zuschläge oder höhere Abschläge bei der Umsatzsteuer $63 \%$ eines Anstiegs inrer Finanzkraft. Länder, die allgemeine Bundesergänzungszuweisungen erhalten, verzeichnen dort einen weiteren Verlust von fast $30 \%$ ihrer zusätzlichen Finanzkraft. Sie unterliegen damit einer Grenzbelastung von insgesamt rund $93 \%$, was gewiss nicht mehr als anreizfreundlich gelten kann.

Das Bild verschlechtert sich gravierend, wenn die Grenzbelastungen nicht auf die Finanzkraft, sondern auf die Steuerkraft der Länder bezogen werden. Als Beispiel dient eine Erhöhung des Lohnsteueraufkommens. Von den Mehreinnahmen erhalten der Bund und das Land jeweils $42,5 \%$ sowie die Gemeinden $15 \%$. Die an die Gemeinden fließende Lohnsteuer wird im Länderfinanzausgleich zu $75 \%$ als Finanzkraft des Landes gewertet. Daher steigt die gemessene Finanzkraft des Landes von $42,5 \%$ auf $53,75 \%$. Das Verhältnis zwischen den für Ausgleichszwecke gemessenen und den faktisch an das Land fließenden Einnahmen aus der Lohnsteuer beträgt $53,75 \%$ zu $42,5 \%$, also ca. $126 \%$. Dieser Multiplikator erhöht die Grenzbelastung eines Landes mit überdurchschnittlicher Finanzkraft von $63 \%$ auf $80 \%$. Für Länder mit unterdurchschnittlicher Finanzkraft, die allgemeine Bundesergänzungszuweisungen erhalten, wächst die Grenzbelastung auf $117 \%$. Nach Finanzausgleich verzeichnen diese Länder bei steigender Lohnsteuerkraft also einen Rückgang ihrer Einnahmen im Landeshaushalt. 
Damit ist das Maximum der Grenzbelastungen aber noch nicht erreicht. Länder, die Gemeindesteuerkraftzuweisungen erhalten, verlieren auch noch $53,5 \%$ des Gemeindeanteils von $15 \%$, also rund $8 \%$ der Lohnsteuermehreinnahmen. Das erhöht die Grenzbelastung bezogen auf den Länderanteil um etwa 19 Prozentpunkte. Unter Berücksichtigung der Ergänzungszuweisungen nach der Gemeindesteuerkraft liegt die Grenzbelastung der betreffenden Länder somit bei ca. 136\%. Solche Grenzbelastungen sind extrem anreizfeindlich. Sie führen gerade finanzschwache Länder in eine Armutsfalle, in der sich eigene Bemühungen zur Erhöhung der Wirtschaftskraft und der Effizienz der Steuerverwaltung nicht mehr lohnen.

Abbildung 6 zeigt die Grenzbelastungen bei der Lohnsteuer differenziert nach den einzelnen Komponenten. Die theoretischen Maximalwerte abstrahieren von den Rückkoppelungseffekten im System des Länderfinanzausgleichs. Ein Anstieg der Finanzkraft eines Landes erhöht auch die durchschnittliche Finanzkraft und damit die Ausgleichsmesszahlen aller Länder. Die effektiven Grenzbelastungen der Länder liegen daher stets unter den theoretischen Werten (Scherf, 2020). Dennoch erreichen sie bei allen Ländern, die Bundesergänzungszuweisungen erhalten, bezogen auf den Landeshaushalt ein konfiskatorisches Niveau.

Diese Berechnung überzeichnet die Grenzbelastungen, weil die Länderhaushalte isoliert betrachtet werden. Länder und Gemeinden erbringen die öffentlichen Leistungen auf ihrem Gebiet gemeinsam, sodass eine integrierte Betrachtung angemessen erscheint. Zwar haben die Länder keinen direkten Zugriff auf die Gemeindesteuern, aber sie können eine Nettobelastung ihres Haushalts über geringere Finanzzuweisungen an die Gemeinden prinzipiell korrigieren. Abbildung 6 zeigt daher auch die gemeinschaftlichen Grenzbelastungen der Länder und Gemeinden. Die Veränderungen, die im Länderfinanzausgleich bei einem Anstieg der Lohnsteuereinnahmen eintreten, werden dabei auf die Summe der Lohnsteueranteile der Länder und Gemeinden, also auf insgesamt 57,5\% der Lohnsteuereinnahmen bezogen. Die so berechneten Grenzbelastungen fallen weiterhin sehr hoch aus und erreichen $96 \%$ bis $99 \%$ bei den Ländern, die Bundesergänzungszuweisungen nach der Gemeindesteuerkraft erhalten. Insofern bleibt es auch auf dieser Basis dabei, dass der heutige Länderfinanzausgleich - wie das Vorgängersystem (Burret et al., 2018) - extrem hohe Grenzbelastungen erzeugt.

Die Grenzbelastungen bei der Lohnsteuer markieren noch keineswegs die Obergrenze der aktuellen Abschöpfungen. Nach der Reform erreichen die Grenzbelastungen bei der Gewerbesteuer ein noch weit höheres Niveau (Scherf, 2020, 13). Bezogen auf den Gesamthaushalt beläuft sich das theoretische Maximum auf fast $122 \%$.
Abbildung 6

\section{Grenzbelastungen bei der Lohnsteuer}

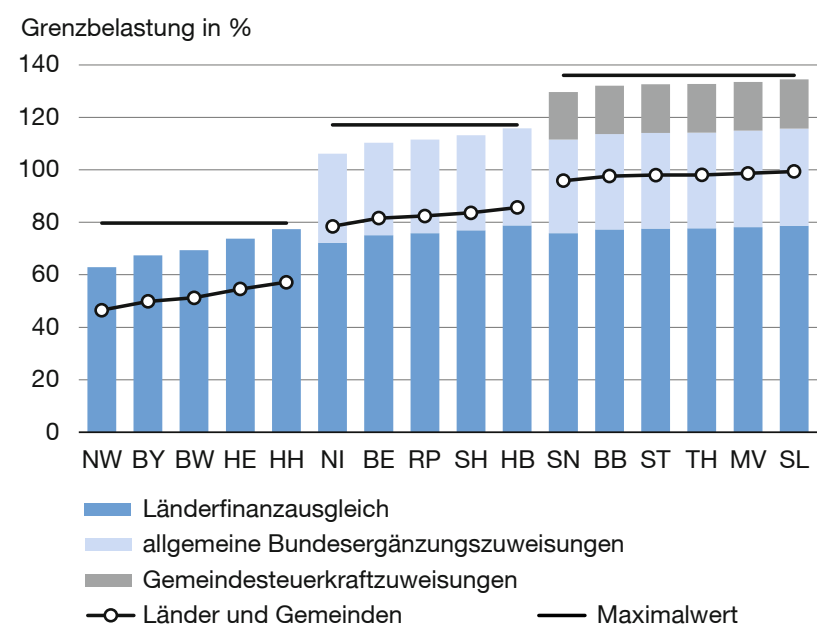

Quelle: eigene Darstellung.

Gegenüber 2019 sorgen die Gemeindesteuerkraftzuweisungen für einen massiven Belastungsanstieg um ca. 60 Prozentpunkte bei den Empfängern. Deren Grenzbelastungen variieren zwischen $116 \%$ (SN) und $120 \%$ (SL) und liegen damit um ca. 20 Prozentpunkte über den Grenzbelastungen bei der Lohnsteuer. ${ }^{3}$

Ein Abbau der Grenzbelastungen ist innerhalb des bestehenden Systems nur erreichbar, wenn das Gewicht der finanzkraftabhängigen Elemente reduziert wird. Ein wichtiger Schritt wäre die Abschaffung der besonders anreizfeindlichen Ergänzungszuweisungen für unterproportionale Gemeindesteuerkraft. Dadurch sinken die theoretischen Grenzbelastungen der Empfängerländer bei der Lohnsteuer deutlich um 19 Prozentpunkte im Landeshaushalt bzw. 14 Prozentpunkte im Gesamthaushalt. Wenn der Bund die Zuweisungen durch eine Erhöhung des Umsatzsteueranteils der Länder kompensiert, halten sich die Umverteilungseffekte in Grenzen. Gemessen am Status quo verlieren die bisherigen Empfänger bis zu 121 Euro pro Einwohner und die anderen Länder gewinnen bis zu 23 Euro pro Einwohner. Die Einbußen sind für die Betroffenen unerfreulich, aber kaum zu beanstanden, denn die Bundesergänzungszuweisungen für unterproportionale Gemeindesteuerkraft haben keinerlei systematische Berechtigung. ${ }^{4}$

3 Büttner und Görbert $(2016,822)$ belegen die extremen Umverteilungseffekte bei Gemeindesteuern durch einen Vergleich der Verbleibsbeträge.

4 Um den Wegfall der Gemeindesteuerkraftzuweisungen zu verbessern, erscheint die Idee naheliegend, die Gemeindesteuerkraft zu $100 \%$ in die Finanzkraft der Länder einzubeziehen. Eine Vollanrechnung würde jedoch die Grenzbelastungen des Landeshaushalts um 6 bis 8 Prozentpunkte erhöhen. Sie steht somit dem Reformziel der Verbesserung der Anreize zur Steigerung der eigenen Wirtschafts- und Steuerkraft diametral entgegen. 


\section{Bundesergänzungszuweisungen als Pauschaltransfers}

Ein Wegfall der Ergänzungszuweisungen für unterproportionale Gemeindesteuerkraft würde immerhin die absoluten Spitzenbelastungen des neuen Systems und die finanzausgleichspolitisch wie verfassungsrechtlich kritischen Übernivellierungseffekte beseitigen. Ihre Abschaffung erscheint auch in kurzer Frist politisch durchsetzbar, wenn sie durch übergangsweise gezahlte Pauschaltransfers abgefedert wird. Dieses Instrument eröffnet aber noch weiter reichende Reformperspektiven. Man könnte auch die allgemeinen Bundesergänzungszuweisungen durch Pauschaltransfers ersetzen und damit die Anreizeffekte des Länderfinanzausgleichs merklich verbessern, ohne seine Verteilungseffekte entscheidend zu schwächen. Dieser Ansatz dürfte zwar stärkere politische Widerstände erzeugen. Die mit geringen Risiken behaftete kleine Reform der Gemeindesteuerkraftzuweisungen könnte aber bei erfolgreichem Verlauf den Boden für eine spätere grundlegende Neugestaltung des Systems bereiten.

Die Idee der Pauschaltransfers basiert auf einer klaren Trennung zwischen Effizienz und Verteilung. Alle finanzkraftabhängigen Bundesergänzungszuweisungen werden abgeschafft und die daraus resultierenden Mindereinnahmen bei der Umstellung vollständig durch Pauschalzahlungen an die betroffenen Länder kompensiert (Kompensations-BEZ). Das Volumen der Bundesergänzungszuweisungen bleibt insgesamt gleich, ist aber nach der Umstellung nominal konstant und vor allem völlig unabhängig von der weiteren Finanzkraftentwicklung. ${ }^{5}$ Diese Maßnahmenkombination bewirkt zunächst einmal keine finanziellen Verschiebungen zwischen den Bundesländern gegenüber dem Status quo. Alle Länder unterliegen aber nur noch der $63 \%$-Belastung durch die Zu- und Abschläge bei der Umsatzsteuer, wenn sich ihre Finanzkraft in Zukunft ändert. Bei der Lohnsteuer erreichen die theoretischen Grenzbelastungen dann nur noch $80 \%$ im Landeshaushalt und $59 \%$ im Gesamthaushalt (vgl. Abbildung 7). Die tatsächlichen Abschöpfungsquoten sind aus den erwähnten Gründen noch etwas niedriger.

Das Interesse an einer verbesserten eigenen Wirtschaftsund Steuerkraft dürfte dadurch steigen. Auch Investitionen in die Steuerverwaltung würden rentabler, wenn ein Land nicht nur die Kosten der Steuerprüfer bezahlen muss, sondern auch die Erträge zu einem größeren Teil vereinnahmen kann. Im Gegensatz zu Reformen mit starken Umverteilungseffekten besteht hier kein Konflikt zwischen kurzfristigen Einbußen und langfristigen Chancen auf Mehreinnah-

5 Die ldee der Reduktion der Grenzbelastungen durch Umstellung der Bundesergänzungszuweisungen auf Pauschaltransfers ist nicht neu, vgl. Huber und Lichtblau (1998); Berthold und Fricke (2007); Büttner et al. (2015).

\section{Abbildung 7 \\ Grenzbelastungen bei der Lohnsteuer im \\ Reformmodell}

Grenzbelastung in \%

100

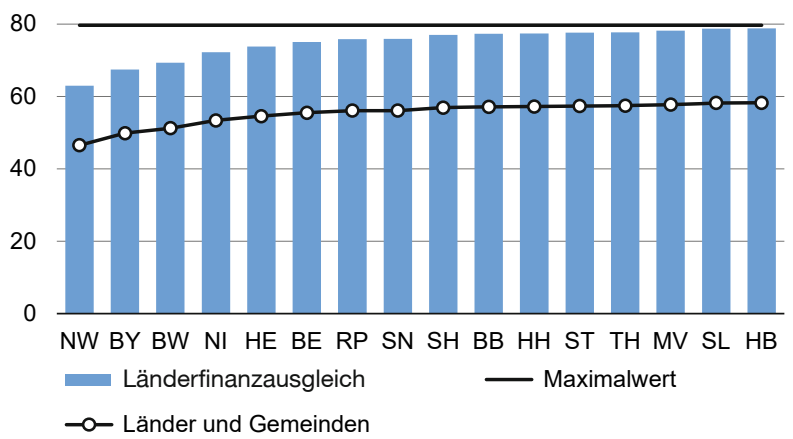

Quelle: eigene Darstellung.

men durch eine wirtschaftsfördernde Standortpolitik. ${ }^{6}$ Die Umstellung würde auch die Systematik des Länderfinanzausgleichs verbessern. Einerseits entfallen die divergenten Bezugsgrößen (Länderfinanzkraft, Gemeindesteuerkraft) und andererseits regelt das Reformmodell den Finanzkraftausgleich auf einer Stufe statt mit drei unterschiedlichen und schlecht aufeinander abgestimmten Instrumenten. Zwar steigt das Volumen der Bundesergänzungszuweisungen für Sonderbedarfe durch die Kompensations-BEZ an, aber im Gegenzug wird der vertikale Bund-LänderAusgleich von der permanenten Aufgabe des horizontalen Länderfinanzausgleichs vollständig befreit.

Es kann durchaus sein, dass sich die Pauschaltransfers auf Dauer politisch schwer rechtfertigen und aufrechterhalten lassen. Aber das ist eher ein Vorteil. Man kann die Pauschaltransfers ausdrücklich als zeitlich befristete Übergangshilfen auf dem Weg zu einem anreizfreundlicheren System deklarieren und sie z. B. nach zehn Jahren auslaufen lassen. Finanzielle Einbußen für die Ländergesamtheit lassen sich dabei vermeiden, wenn parallel zum kontinuierlichen Abbau der Kompensations-BEZ der Umsatzsteueranteil der Länder entsprechend angehoben wird, was allen Ländern mit gleichen Pro-Kopf-Beträgen zugute käme.

Freilich können die Finanzkraftdifferenzen zwischen den Ländern nach der Umstellung - gefördert durch das Abschmelzen und Auslaufen der Pauschaltransfers - so stark anwachsen, dass die Gleichwertigkeit der Lebensverhält-

6 Ein lascher Steuervollzug kann auch der versteckten Subventionierung der heimischen Wirtschaft dienen. Dann motivieren finanzielle Anreize nicht ohne Weiteres zu einer verstärkten Steuerprüfung (Troost, 2016, 665). 
nisse gefährdet erscheint. Vorbeugen kann man diesem Problem durch einen alternativen Umgang mit den Pauschaltransfers. Statt der Umwidmung in Umsatzsteueranteile wäre auch eine Verwendung für Zweckzuweisungen zur Förderung strukturschwacher Regionen denkbar. Dabei sollten nur Maßnahmen zur Beseitigung von Infrastrukturlücken dotiert werden, an deren Finanzierung sich die Länder beteiligen. Andernfalls besteht die Gefahr einer ineffizienten oder konsumtiven Mittelverwendung. Bei Erfolg der getroffenen Maßnahmen rücken die Finanzkraftpositionen vor Finanzausgleich zusammen und der notwendige Umfang des horizontalen Ausgleichs fällt entsprechend kleiner aus.

Selbst bei investivem Mitteleinsatz ist nicht ausgemacht, dass finanzschwache Länder eine Finanzkraft vor Finanzausgleich realisieren, die in Verbindung mit dem linearen Ausgleichstarif eine angemessene Mindestfinanzkraft nach Finanzausgleich garantiert. ${ }^{7}$ Im Reformmodell lässt sich hierfür aber eine Bremsfunktion einbauen. Wenn finanzschwache Länder bei planmäßiger Rückführung der Pauschaltransfers die Mindestfinanzkraft unterschreiten, kann man den Abbau aussetzen bzw. sein Tempo verlangsamen. Der Preis wäre eine entsprechend geringere Überführung frei werdender Bundesmittel in die Umsatzsteuerverteilung bzw. in die Infrastrukturförderung.

\section{Fazit}

Der Länderfinanzausgleich ist mit der Reform weder einfacher noch gerechter geworden. Die Verlagerung des horizontalen Ausgleichs in die Umsatzsteuerverteilung hat die Intransparenz eher gesteigert. Auch die Ausweitung der Bundesergänzungszuweisungen ist kein Fortschritt, sondern dient allein der weiteren oder zusätzlichen Dotierung spezieller Länderinteressen. Insgesamt bleiben die Defekte des Länderfinanzausgleichs nicht nur erhalten, sondern werden teilweise noch verstärkt. Das gilt vor allem für die Grenzbelastungen, die bei den Empfängerländern der Gemeindesteuerkraftzuweisungen ein rekordverdächtiges Niveau erreichen.

Im präsentierten Modell zur Verbesserung des Länderfinanzausgleichs werden die Gemeindesteuerkraftzuweisun-

7 Man könnte den Ausgleichsatz in diesem Fall über das Normalniveau anheben und bei Annäherung der Finanzkraftpositionen wieder absenken (Scherf, 2001). Allerdings würden die Grenzbelastungen dadurch temporär steigen. gen und später auch die allgemeinen Bundesergänzungszuweisungen auf Pauschaltransfers umgestellt. Sie entsprechen zunächst der länderspezifischen Summe der heutigen Zuweisungen, werden aber schrittweise abgeschmolzen und laufen schließlich nach einer Übergangszeit von z.B. zehn Jahren aus. Die freigesetzten Bundesmittel fließen kontinuierlich in den Umsatzsteueranteil der Länder oder in die Infrastrukturförderung. Der Finanzkraftausgleich wird damit auf seine horizontale Dimension reduziert.

Per saldo sorgt das Modell für eine erhebliche Reduktion der Grenzbelastungen bei zunächst stabiler Finanzkraftverteilung. Erst langfristig erreicht die Nivellierung der Finanzkraft durch Kürzung der Pauschaltransfers ein niedrigeres Niveau. Inwieweit das politisch durchsetzbar ist, muss hier offen bleiben. Immerhin bietet das Modell eine hohe Gestaltungsfreiheit durch zeitliche Streckung des Umstellungsprozesses, ohne seinen entscheidenden Vorteil der sofort und deutlich sinkenden Grenzbelastungen deshalb zu verlieren. Damit eröffnet sich ein Weg zur systematischen statt kosmetischen Korrektur des Länderfinanzausgleichs.

\section{Literatur}

Berthold, N., und H. Fricke (2007), Der Länderfinanzausgleich - wie seh schadet er, wie sollte er reformiert werden?, Wirtschaftswissenschaftliche Beiträge des Lehrstuhls für Volkswirtschaftslehre.

BMF (Bundesministerium der Finanzen) (2020), Erste Verordnung zur Durchführung des Finanzausgleichsgesetzes im Ausgleichsjahr 2020, Bundesrat Drucksache, 62/20.

Büttner, T., und T. Görbert (2016), Neuregelung des bundesstaatlichen Finanzausgleichs, Wirtschaftsdienst, 96(11), 818-824.

Büttner, T., J. Hey, A. Weichenrieder und H. Zimmermann (2015), Reform des Finanzausgleichs: Eigenverantwortung stärken und Transparenz verbessern, Wirtschaftsdienst, 95(10), 655-659.

Burret, H. T., Y. Bury und L. Feld (2018), Grenzabschöpfungsraten im deutschen Finanzausgleich, List Forum, 44/2018, 1-22.

Glinka, P., und T. Lenk (2015), Steuerzuordnung nach der Wirtschaftskraft - gut für den bundesstaatlichen Finanzausgleich, Wirtschaftsdienst, 95(9), 619-626.

Heinemann, F. (2016), Länder auf dem Weg in die vollständige Alimentation, ifo Schnelldienst, 69(24), S. 13-15.

Huber, B., und K. Lichtblau (1998), Konfiskatorischer Finanzausgleich verlangt eine Reform, Wirtschaftsdienst, 78(3), 142-147.

Scherf, W. (2001), Ein Vorschlag für einen gerechten und effizienten Länderfinanzausgleich, Wirtschaftsdienst, 81(4), S. 227-234.

Scherf, W. (2020), Grenzbelastungen im Länderfinanzausgleich, Finanzwissenschaftliche Arbeitspapiere, 103.

Troost, A. (2016), Nimmt der Länderfinanzausgleich den Anreiz zum Steuervollzug?, Wirtschaftsdienst, 96(9), 660-666.

Weiß, R., und W. Münzenmaier (2014), Auswirkungen einer Lohnsteuerzerlegung nach Arbeitsort im Länderfinanzausgleich, Wirtschaftsdienst, 94(10), 732-739.

Title: Federal State Fiscal Equalisation 2020: New Scheme - Old Problems

Abstract: The new federal state fiscal equalisation will be implemented for the first time in 2020. Its functioning and effects are presented and illustrated in detail. Compared to the old system, there are large formal differences but only minor real differences. The reform has not eliminated existing deficits, but rather exacerbated them. The author proposes a model for a further reform of the federal state fiscal equalisation to improve the incentive effects.

JEL Classification: $H 7, H 77$ 